\title{
Regular Composition for Slice-Regular Functions of Quaternionic Variable
}

\author{
Fabio Vlacci
}

\begin{abstract}
In general (see e.g. Cartan in Elementary Theory of Analytic Functions of One or Several Complex Variables, 1963), given two (formal) power series $g(x)=b_{0}+x b_{1}+\cdots+x^{n} b_{n}+\cdots$ and $f(x)=x a_{1}+\cdots+x^{n} a_{n}+\cdots$ (with $\left.a_{0}=f(0)=0\right)$ it is well known that the composition of $g$ with $f$, in symbols $g(f(x))$, is a formal power series when coefficients $a_{j}$ and $b_{k}$ are taken in a commutative field. Furthermore, if the constant term $a_{0}$ of the power series $f$ is not 0 , the existence of the composition $g(f(x))$ has been an open problem for many years and only recently has received some partial answers (see Gan and Knox in Int. J. Math. Math. Sci. 30:761-770, 2002). The notion of slice-regularity, recently introduced by Gentili and Struppa (Adv. Math. 216:279-301, 2007), for functions in the non-commutative division algebra $\mathbb{H}$ of quaternions guarantees their quaternionic analyticity but the non-commutativity of the product in $\mathbb{H}$ requires special attention even to define their multiplication (see also Gentili and Stoppato in Michigan Math. J. 56:655-667, 2008). In this paper we face the problem of defining the (sliceregular) composition $g \odot f$ of two slice-regular functions $f, g$; this turns out to be defined as an extension of the standard composition $g \circ f$ of functions in a noncommutative setting which takes into account a non-commutative version of Bell polynomials and a generalization of the Faà di Bruno Formula.
\end{abstract}

\section{Introduction to Slice-Regularity in $\mathbb{H}$}

We recall that the algebra of quaternions $\mathbb{H}$ consists of numbers $x_{0}+i x_{1}+j x_{2}+k x_{3}$ where $x_{l}$ is real $(l=0, \ldots, 3)$, and $i, j, k$, are imaginary units (i.e. their square equals -1 ) such that $i j=-j i=k, j k=-k j=i$, and $k i=-i k=j$. In this way, $\mathbb{H}$ can be considered as a vector space over the real numbers of dimension 4 . Given a generic element $q=x_{0}+i x_{1}+j x_{2}+k x_{3}$ of $\mathbb{H}$ we define in a natural fashion its conjugate $\bar{q}=x_{0}-i x_{1}-j x_{2}-k x_{3}$, and its square norm $|q|^{2}=q \bar{q}=\sum_{k \geq 0}^{3} x_{k}^{2}$. The set $\mathbb{S}=\left\{q \in \mathbb{H}: q^{2}=-1\right\}$ will be referred to as the sphere of imaginary units of $\mathbb{H}$.

\footnotetext{
F. Vlacci $(\bowtie)$

Department of Mathematics “U. Dini”, University of Florence, Viale Morgagni 67/A,

50134 Florence, Italy

e-mail: vlacci@math.unifi.it

G. Gentili et al. (eds.), Advances in Hypercomplex Analysis, Springer INdAM Series 1,

DOI 10.1007/978-88-470-2445-8_9, (C) Springer-Verlag Italia 2013
} 
The following is immediate and yet important

Proposition 1 For any non-real quaternion $w$, there exist, and are unique, $x, y \in \mathbb{R}$ with $y>0$, and an imaginary unit $I_{w}$ such that $w=x+y I_{w}$.

Definition 1 Given any imaginary unit $I$, the set $\mathbb{R}+\mathbb{R} I$ will be denoted by $L_{I}$.

Notice that after identifying the imaginary unit $I_{w}$ in $\mathbb{H}$ with the imaginary unit $i$ of $\mathbb{C}$, the set $L_{I_{w}}$ may be considered as a complex plane in $\mathbb{H}$ passing through 0,1 and $w$. In this way, $\mathbb{H}$ can be obtained as an infinite union of complex planes (which will be also called slices).

Definition 2 If $\Omega$ is a domain in $\mathbb{H}$, a real differentiable function $f: \Omega \rightarrow \mathbb{H}$ is said to be slice-regular if, for every $I \in \mathbb{S}_{\mathbb{K}}$, its restriction $f_{I}$ to the complex line $L_{I}=\mathbb{R}+\mathbb{R} I$ passing through the origin and containing 1 and $I$ is holomorphic on $\Omega \cap L_{I}$.

In particular any slice-regular function is $C^{\infty}$ in $\mathbb{B}(0, R) .{ }^{1}$ Actually, something more is proven in [5]:

Theorem 1 A function $f: B=B(0, R) \rightarrow \mathbb{K}$ is regular if, and only if, it has a series expansion of the form

$$
f(q)=\sum_{n=0}^{+\infty} q^{n} \frac{1}{n !} \frac{\partial_{C}^{n} f}{\partial x^{n}}(0)
$$

converging in $B$. In particular if $f$ is regular then it is $C^{\infty}$ in $B$.

For an introductory survey on slice-regular functions we refer the interested reader to [7].

Let $f(q)=\sum_{n=0}^{+\infty} q^{n} a_{n}$ be a given slice-regular function whose associated quaternionic power series has radius of convergence $R>0$ and consider the quaternionic power series with real coefficients $\sum_{n=0}^{+\infty} q^{n}\left|a_{n}\right|$; it also has radius of convergence $R$, because, according to Hadamard's Formula (see e.g. [1]),

$$
R= \begin{cases}1 / \lim \sup _{n \rightarrow \infty}\left|a_{n}\right|^{1 / n} & \text { if the limsup is finite and different from } 0 \\ 0 & \text { if } \limsup _{n \rightarrow \infty}\left|a_{n}\right|^{1 / n}=+\infty \\ +\infty & \text { if } \limsup _{n \rightarrow \infty}\left|a_{n}\right|^{1 / n}=0\end{cases}
$$

Therefore the function

$$
z \mapsto \sum_{n=0}^{+\infty} q^{n}\left|a_{n}\right|
$$

\footnotetext{
${ }^{1}$ This smoothness is considered with respect to the so called Cullen derivative $\partial_{C}$ (see also [2, 5]) which is well-defined for slice-regular functions as follows: if $f(q)=\sum_{n=0}^{+\infty} q^{n} a_{n}$ then $\partial_{C} f(q):=$ $\sum_{n=1}^{+\infty} n q^{n-1} a_{n}$.
} 
(denoted by $\left.f_{a b s}(z)\right)$ is a slice-regular function in $B(0, R)$ with the property that for any $I \in \mathbb{S}$, we have $f_{a b s}\left(L_{I}\right) \subset L_{I}$.

\section{Non-commutative Bell Polynomials and Slice-Regular Composition}

Let $k=\left(k_{1}, k_{2}, \ldots, k_{n}\right) \in \mathbb{N}^{n}$ be a given multiindex; we set

$$
\begin{aligned}
k ! & :=k_{1} ! k_{2} ! \cdots k_{n} ! \\
|k| & :=k_{1}+k_{2}+\cdots+k_{n} \\
\|k\| & :=k_{1}+2 k_{2}+\cdots+n k_{n} .
\end{aligned}
$$

Then for $n \geq 1$, we define

$$
B_{n}\left(y_{1}, \ldots, y_{n}\right):=\sum_{\|k\|=n} \frac{n !}{k !}\left(\frac{y_{1}}{1 !}\right)^{k_{1}} \cdot\left(\frac{y_{2}}{2 !}\right)^{k_{2}} \cdots\left(\frac{y_{n}}{n !}\right)^{k_{n}}
$$

where $y_{j}$ are elements of a commutative algebra; we observe that these polynomials satisfy the recursive equation

$$
B_{n+1}=\sum_{k=0}^{n}\left(\begin{array}{l}
n \\
k
\end{array}\right) B_{n-k} y_{k+1}
$$

with the initial condition $B_{0}=1$. These polynomials are called Bell polynomials. If one decomposes $B_{n}$ into its homogeneous ${ }^{2}$ parts $B_{n, d}$ (with $d=1,2, \ldots, n$ ), one can write

$$
B_{n}=\sum_{d=1}^{n} B_{n, d}
$$

and obtain

$$
B_{n, d}\left(y_{1}, \ldots, y_{n}\right):=\sum_{\|k\|=n,|k|=d} \frac{n !}{k !}\left(\frac{y_{1}}{1 !}\right)^{k_{1}} \cdot\left(\frac{y_{2}}{2 !}\right)^{k_{2}} \cdots\left(\frac{y_{n}}{n !}\right)^{k_{n}} .
$$

The homogeneous polynomials $B_{n, d}$ appear in the expression of the $n$-th derivative of the chain rule, namely

Proposition 2 (Faà di Bruno Formula) If $h=g \circ f$, then

$$
h^{(n)}(x)=\sum_{d=1}^{n} B_{n, d}\left(f^{\prime}(x), f^{\prime \prime}(x), \ldots, f^{(n)}(x)\right) \cdot g^{(d)}(f(x)) .
$$

\footnotetext{
${ }^{2}$ A polynomial $B_{n, d}$ is homogeneous of degree $d$ if

$$
B_{n, d}\left(\lambda y_{1}, \lambda y_{2}, \ldots, \lambda y_{n}\right)=\lambda^{d} B_{n, d}\left(y_{1}, y_{2}, \ldots, y_{n}\right)
$$
}

for any $\lambda$. 
In [8], the authors extend the notion of Bell polynomials to the setting of a noncommutative algebra $\mathscr{A}$ with unit and obtain their explicit expressions, namely

$$
\widetilde{B}_{n, d}:=\sum_{n_{2}, \ldots, n_{d}=1}^{n}\left(\begin{array}{c}
n-1 \\
n_{2}
\end{array}\right)\left(\begin{array}{c}
n_{2}-1 \\
n_{3}
\end{array}\right) \cdots\left(\begin{array}{c}
n_{d-1}-1 \\
n_{d}
\end{array}\right) y_{n_{d}} y_{n_{d-1}-n_{d}} \cdots y_{n_{2}-n_{3}} y_{n-n_{2}}
$$

for $n \geq d \geq 2$ and $\widetilde{B}_{0}=1, \widetilde{B}_{1}=y_{1}$. It is then natural to consider

$$
\widetilde{B}_{n}=\sum_{d=1}^{n} \widetilde{B}_{n, d}
$$

These polynomials also satisfy the analogous of Eq. (1); in particular, because of the non-commutativity of multiplication, if instead of

$$
\widetilde{B}_{n+1}=\sum_{k=0}^{n}\left(\begin{array}{l}
n \\
k
\end{array}\right) \widetilde{B}_{n-k} y_{k+1}
$$

(with the initial condition $\widetilde{B}_{0}=1$ ) one considers the condition

$$
\widetilde{B}_{n+1}=\sum_{k=0}^{n}\left(\begin{array}{l}
n \\
k
\end{array}\right) y_{k+1} \widetilde{B}_{n-k}
$$

then

$$
\widetilde{B}_{n, d}:=\sum_{n_{2}, \ldots, n_{d}=1}^{n}\left(\begin{array}{c}
n-1 \\
n_{2}
\end{array}\right)\left(\begin{array}{c}
n_{2}-1 \\
n_{3}
\end{array}\right) \cdots\left(\begin{array}{c}
n_{d-1}-1 \\
n_{d}
\end{array}\right) y_{n-n_{2}} y_{n_{2}-n_{3}} \cdots y_{n_{d-1}-n_{d}} y_{n_{d}}
$$

for $n \geq d \geq 2$ and $\widetilde{B}_{0}=1, \widetilde{B}_{1}=y_{1}$. Moreover the expressions of $\widetilde{B}_{n, d}$ can be also inductively obtained by means of a derivation ${ }^{3} D$ plus the multiplication from the left of an element of $\mathscr{A}$. In fact, if we adopt the notation $D(y)=y^{\prime}$ and $D\left(y^{(n-1)}\right)=y^{(n)}$, the (non-commutative) Bell polynomials which satisfy (3) are uniquely determined by

$$
\widetilde{B}_{n+1}\left(y, y^{\prime}, y^{\prime \prime}, \ldots, y^{(n)}\right)=(D+y) \widetilde{B}_{n}\left(y, y^{\prime}, y^{\prime \prime}, \ldots, y^{(n-1)}\right), \quad \widetilde{B}_{0}=1,
$$

where

$$
(D+y) \widetilde{B}:=D(\widetilde{B})+y B
$$

\footnotetext{
${ }^{3} \mathrm{~A}$ derivation is an endomorphism

$$
D: \mathscr{A} \rightarrow \mathscr{A}
$$

of an associative (generally non-commutative) algebra $\mathscr{A}$ with unit 1 , such that $D\left(y_{1} \cdot y_{2}\right)=D\left(y_{1}\right)$. $y_{2}+y_{1} \cdot D\left(y_{2}\right)$. In particular $D(1)=0$.
} 
for (non-commutative) Bell polynomials which satisfy (4) one has to consider the action of $\underset{\widetilde{B}}{(}(D+y)$ on the right. Thus one immediately observes that $\widetilde{B}_{n, 1}\left(y_{1}, y_{2}, \ldots, y_{n}\right)=$ $y_{n}$ and $\widetilde{B}_{n, n}\left(y_{1}, y_{2}, \ldots, y_{n}\right)=y_{1}^{n}$; more in general

$$
\begin{array}{ll}
\widetilde{B}_{0} & =1 \\
\widetilde{B}_{1}\left(y_{1}\right) & =y_{1} \\
\widetilde{B}_{2}\left(y_{1}, y_{2}\right) & =y_{2}+y_{3}+\underbrace{y_{2} y_{1}+2 y_{1} y_{2}}_{\widetilde{B}_{3,2}\left(y_{1}, y_{2}, y_{3}\right)}+\underbrace{\widetilde{B}_{3}\left(y_{1}, y_{2}, y_{3}\right)}_{\widetilde{B}_{4,2}\left(y_{1}, y_{2}, y_{3}\right)}=y_{\widetilde{B}_{4,3}\left(y_{1}, y_{2}, y_{3}, y_{4}\right)}^{y_{3} y_{1}+3 y_{2}^{2}+3 y_{1} y_{3}}+\underbrace{y_{2} y_{1}^{2}+2 y_{1} y_{2} y_{3}+3 y_{1}^{2} y_{2}}+y_{1}^{4} \\
\widetilde{B}_{4}\left(y_{1}, y_{2}, y_{3}, y_{4}\right) & =y_{4}
\end{array}
$$

In any case, it turns out that

$$
\begin{aligned}
\left|\widetilde{B}_{n}\left(y_{1}, \ldots, y_{n}\right)\right| & \leq \sum_{d=1}^{n}\left|\widetilde{B}_{n, d}\left(y_{1}, \ldots, y_{n}\right)\right| \\
& \leq \sum_{d=1}^{n} B_{n, d}\left(\left|y_{1}\right|, \ldots,\left|y_{n}\right|\right)=B_{n}\left(\left|y_{1}\right|, \ldots,\left|y_{n}\right|\right) .
\end{aligned}
$$

Assume now two slice-regular functions $f(q)=\sum_{n=0}^{+\infty} q^{n} a_{n}$ and $g(q)=\sum_{n=0}^{+\infty} q^{n} b_{n}$ are given. Since $f(0)=a_{0}$ and $\partial_{C}^{n} f(0)=n ! a_{n}$, in analogy to (2), we define $g^{\odot} f(q)$ to be the slice regular function

$$
g^{\odot} f(q):=\sum_{n=0}^{+\infty} q^{n} c_{n}
$$

whose coefficients are given by

$$
c_{0}=g\left(a_{0}\right), \quad c_{n}=\frac{1}{n !} \sum_{d=1}^{n} \widetilde{B}_{n, d}\left(a_{1}, 2 ! a_{2}, \ldots, n ! a_{n}\right) \cdot g^{(d)}\left(a_{0}\right), \quad n \geq 1,
$$

with $\widetilde{B}_{n, d}$ the corresponding non-commutative Bell polynomial. In particular, if $f(0)=0=a_{0}$, then we have

$$
c_{n}=\frac{1}{n !} \sum_{d=1}^{n} d ! \widetilde{B}_{n, d}\left(a_{1}, 2 ! a_{2}, \ldots, n ! a_{n}\right) \cdot b_{d} .
$$

Clearly, from (5), for $n \geq 1$, one has

$$
\begin{aligned}
\left|c_{n}\right| & \leq \frac{1}{n !} \sum_{d=1}^{n}\left|\widetilde{B}_{n, d}\left(a_{1}, 2 ! a_{2}, \ldots, n ! a_{n}\right)\right| \cdot\left|g^{(d)}\left(a_{0}\right)\right| \\
& \leq \frac{1}{n !} \sum_{d=1}^{n} B_{n, d}\left(\left|a_{1}\right|, 2 !\left|a_{2}\right|, \ldots, n !\left|a_{n}\right|\right) \cdot\left|g^{(d)}\left(a_{0}\right)\right|
\end{aligned}
$$


and the last sum of the right-hand side in the previous inequality corresponds to the coefficients of the power expansion of $g_{a b s} \circ f_{a b s}$. In other words, we have the following

Proposition 3 Given two slice-regular functions $f(q)=\sum_{n=0}^{+\infty} q^{n} a_{n}$ and $g(q)=$ $\sum_{n=0}^{+\infty} q^{n} b_{n}$ in $\mathbb{H}$, if the composition $g_{a b s} \circ f_{a b s}$ of the corresponding associated abspower series exists and have radius of convergence $R$ then it is possible to define the slice-regular function $g^{\odot} f$ for $q$ such that $|q|<R$ in terms of the power series

$$
g^{\odot} f(q)=\sum_{n=0}^{+\infty} q^{n} c_{n}
$$

where the coefficients are given in (6).

Proof Indeed, the coefficients given in (6) satisfy inequality (7) and this guarantees the convergence of the series $\sum_{n=0}^{+\infty} q^{n} c_{n}$ for any $q$ such that $|q|<R$. Finally, because of Theorem 1, the function

$$
q \mapsto \sum_{n=0}^{+\infty} q^{n} c_{n}
$$

is slice-regular.

Remark 1 The functions $f_{a b s}$ and $g_{a b s}$ have power series expansions whose coefficients are real numbers, so all the classical results in [1] (and more recent ones in [3]) which provide sufficient conditions for the existence of the composition of formal power series with coefficients in a commutative field apply. In particular the composition $g_{a b s} \circ f_{a b s}$ exists if $a_{0}=0$.

Remark 2 Since apparently associativity of the product is never applied, a similar result should hold true also for slice-regular functions of octonionic variable (see [6]).

Acknowledgements The author has been partially supported by Progetto MIUR di Rilevante Interesse Nazionale Proprietà geometriche delle varietà reali e complesse and by G.N.S.A.G.A (gruppo I.N.d.A.M).

\section{References}

1. Cartan, H.: Elementary Theory of Analytic Functions of One or Several Complex Variables. Addison-Wesley, Reading (1963)

2. Cullen, C.G.: An integral theorem for analytic intrinsic functions on quaternions. Duke Math. J. 32, 139-148 (1965)

3. Gan, X.-X., Knox, N.: On composition of formal power series. Int. J. Math. Math. Sci. 30, 761-770 (2002) 
4. Gentili, G., Stoppato, C.: Zeros of regular functions and polynomials of a quaternionic variable. Mich. Math. J. 56, 655-667 (2008)

5. Gentili, G., Struppa, D.C.: A new theory of regular functions of a quaternionic variable. Adv. Math. 216, 279-301 (2007)

6. Gentili, G., Struppa, D.C.: Regular functions on the space of Cayley numbers. Rocky Mt. J. Math. 40(1), 225-241 (2010)

7. Gentili, G., Stoppato, C., Struppa, D.C., Vlacci, F.: Recent developments for regular functions of a hypercomplex variable. In: Hypercomplex Analysis. Trends in Mathematics, pp. 165-186. Birkhäuser, Basel (2009)

8. Schimming, R., Zagloul Rida, S.: Non commutative Bell polynomials. Int. J. Algebra Comput. 6(5), 635-644 (1996) 